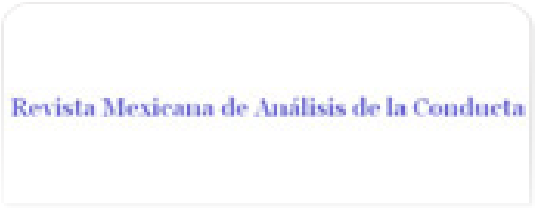

Revista Mexicana de Análisis de la Conducta ISSN: 0185-4534

editora@rmac-mx.org

Sociedad Mexicana de Análisis de la Conducta México

Jiménez Rodríguez, David; Guevara Benítez, Yolanda

Comparación de dos estrategias de intervención en interacciones madre-hijo. Su relación con el rendimiento escolar

Revista Mexicana de Análisis de la Conducta, vol. 34, núm. 2, diciembre, 2008, pp. 221-246

Sociedad Mexicana de Análisis de la Conducta

Guadalajara, México

Disponible en: http://www.redalyc.org/articulo.oa?id=59311115006

- Cómo citar el artículo

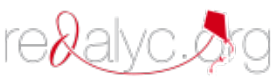

- Número completo

- Más información del artículo

- Página de la revista en redalyc.org

Sistema de Información Científica

Red de Revistas Científicas de América Latina, el Caribe, España y Portugal Proyecto académico sin fines de lucro, desarrollado bajo la iniciativa de acceso abierto 


\title{
COMPARACIÓN DE DOS ESTRATEGIAS DE INTERVENCIÓN EN INTERACCIONES MADRE-HIJO. SU RELACIÓN CON EL RENDIMIENTO ESCOLAR ${ }^{1}$
}

\author{
COMPARISON OF TWO INTERVENTION STRATEGIES \\ IN THE MOTHER-CHILD INTERACTION. ITS RELATIONSHIP \\ WITH SCHOOL PERFORMANCE \\ DAVID JIMÉNEZ RODRÍGUEZ Y YOLANDA GUEVARA BENÍTEZ \\ UNIVERSIDAD NACIONAL AUTÓNOMA DE MÉXICO
}

\begin{abstract}
RESUMEN
El objetivo del presente estudio fue aplicar estrategias de intervención para mejorar las interacciones en díadas madre-hijo, como una manera de mejorar el desempeño académico y la conducta en el aula de alumnos de primer grado, de estrato sociocultural bajo. Se comparó el nivel de efectividad de dos estrategias, cada una llevada a cabo en 20 sesiones de dos horas semanales, a través de la comparación de tres grupos homogéneos de 15 díadas cada uno: el Grupo A recibió entrenamiento para mejorar interacciones diádicas, en el Grupo B se entrenó exclusivamente a las madres de familia para mejorar sus prácticas de crianza, el Grupo $\mathrm{C}$ no recibió entrenamiento simultáneo. Se evaluó la efectividad de las estrategias midiendo sus efectos sobre tres variables dependientes: las prácticas de crianza reportadas por madres e hijos, el rendimiento académico (promedio escolar) y la conducta de los alumnos en el aula, antes y después de la intervención. Los mejores efectos se observaron en el Grupo A, considerando las tres variables dependientes. Se discuten las implicaciones, aportaciones y limitaciones del estudio.
\end{abstract}

1. Los autores son profesores de la Facultad de Estudios Superiores, Iztacala. Universidad Nacional Autónoma de México.Av. de los Barrios no. 1, Los Reyes, Iztacala, Tlalnepantla, Estado de México. C. P. 54090. vigos9634@hotmail.com, yolaguevara@hotmail.com

Recibido: 6 de Junio, 2008. Revisado: 9 de Noviembre 9, 2008. Aceptado: 30 de Diciembre, 2008. 
Palabras clave: interacciones diádicas, estilos de crianza, rendimiento académico, conducta inadecuada, educación básica.

\begin{abstract}
The objective of the present research was to apply intervention strategies to improve the interactions of the mother-child dyad, as a way to improve the academic performance and the behavior in first grade students of the low socio-cultural stratum. The effectiveness of two strategies was compared through the comparison of three homogenous groups composed of 15 dyads each. Group A received training to improve dyadic interactions; in Group B exclusively mothers were trained to improve their raising practices; Group C did not receive training at all. Each strategy was applied in 20 two-hour sessions per week. The effectiveness of the strategies was evaluated measuring its effects in three dependent variables: raising practices reported by mothers and children, academic performance (scholar average), and children's behavior in the classroom before and after the intervention. The best results were observed in Group A, considering the three dependent variables. The implications, contributions, and limitations of the study are discussed.

Key words: dyadic mother-child interactions, raising styles, academic performance, classroom behavior, basic education.
\end{abstract}

Entre los planteamientos de la psicología contemporánea se reconoce que es la familia quien puede promover el desarrollo personal y social en los niños, donde los padres ponen en juego estilos de crianza que determinan en gran medida el tipo de relación padre-hijo, así como los niveles de desarrollo psicológico infantil en diversas habilidades y competencias. Dentro de esta disciplina, el contexto familiar se concibe como un sistema que incluye vías de mutua influencia, directa e indirecta, entre sus integrantes. Los estilos de crianza paternos y los patrones de interacción familiar tienen influencia prácticamente en todos los ámbitos de la vida de un individuo en desarrollo: en sus habilidades conductuales y aspectos de personalidad, en sus formas de interacción con la comunidad, e incluso en el nivel de éxito o fracaso en actividades escolares y productivas (Jiménez y Guevara, 2008).

El bajo rendimiento escolar de un alumno y las interacciones que se viven dentro de su hogar se toman muchas veces como aspectos desvinculados, dado que escuela y hogar son ambientes separados y con frecuencia ajenos entre sí. Sin embargo, desde las diferentes corrientes psicológicas se reconoce que el papel que juegan los progenitores dentro de la familia es fundamental para el desarrollo adecuado de cualquier individuo y particularmente 
para su formación académica. Son muchos los estudios que han comprobado que los estilos de crianza y las interacciones familiares desfavorables afectan negativamente el desempeño académico y social de los niños.

La importancia social de este tema radica en que uno de los problemas más relevantes en nuestro país es el índice de bajo rendimiento escolar en estudiantes de nivel primaria. Según datos del Instituto Nacional para la Evaluación Educativa (INEE, 2004, 2006), sólo el 37\% de los alumnos que terminan la primaria muestra un nivel satisfactorio en habilidades lectoras, y en matemáticas dicha proporción es de sólo el 13\%; al comparar a los alumnos considerando el contexto sociocultural (incluyendo lo que el INEE ubica como "capital cultural de las familias") se encontró que este factor explica aproximadamente el $68 \%$ de las diferencias registradas en el aprendizaje de los alumnos, y que en los contextos socioculturales catalogados como "muy desfavorables" la gran mayoría de los alumnos (más del $80 \%$ ) obtuvo niveles académicos insatisfactorios. Esta situación puede llevar al fracaso escolar de amplios sectores de nuestra sociedad.

En ese contexto, se hace necesario que la psicología educativa mexicana desarrolle líneas de investigación que permitan estudiar la relación que guardan los estilos de crianza y las interacciones familiares con el rendimiento escolar, revisando planteamientos teóricos y hallazgos de investigaciones previas, confirmando o refutando esos hallazgos en poblaciones mexicanas, y diseñando estrategias de intervención para mejorar las prácticas de crianza, las interacciones dentro del seno familiar y el desempeño académico de niños mexicanos en situaciones de riesgo.

Las interacciones familiares han sido abordadas desde varios enfoques psicológicos, utilizando términos propios de su tendencia teórica-metodológica. En el presente trabajo se hace referencia a los planteamientos de autores con un enfoque cognitivo-conductual que hacen uso del término estilos de crianza (Lipsitt y Reese, 1983; McKinney, 1981), así como de autores que desde el enfoque conductual manejan términos como interacción padreshijos, interacción diádica y prácticas de crianza (Bijou y Baer, 1982; Guevara y Mares, 1995; Kantor, 1980; Ortega, 1994).

Los hallazgos de las investigaciones relativas a los estilos de crianza permiten tener un panorama general de una serie de factores que propician ciertos estilos, y de la influencia que éstos tienen sobre el desarrollo psicológico infantil, especialmente en variables como el rendimiento académico, el nivel de adaptación social en la escuela y la familia, o el nivel de auto-estima (v. g. Aguilar, Valencia, Martínez, Romero y Lemus, 2004; Lambord, Mounts, Steinberg y Dornbusch, 1991; Steinberg, Lamborn, Darling, Mounts y Dornbusch, 1994). Tales autores ubican cuatro estilos de crianza principales y los caracterizan de la manera siguiente: 
Estilo democrático. Caracteriza a los padres que pueden delimitar reglas dentro del hogar y transmitirlas a sus hijos, haciéndoles saber cuando no hacen lo correcto. Este tipo de padres atiende a las necesidades de sus hijos sin romper las reglas y teniendo una comunicación con ellos para conocer su punto de vista, y juntos establecer acuerdos.

Estilo permisivo. Caracteriza a los padres tolerantes en extremo, que autorizan todo a sus hijos; acuden ante la menor demanda de atención, se oponen a impartir castigos o señalamientos verbales y ceden a la menor insistencia.

Estilo negligente. Caracteriza a los padres tolerantes en extremo, que permiten que sus hijos se comporten como quieran, pero a diferencia de los de estilo permisivo, estos padres no acuden ante las demandas de atención, tampoco imponen castigos y no establecen intercambios o negociaciones con sus hijos.

Estilo autoritario. Caracteriza a los padres sumamente estrictos e intransigentes, que exigen obediencia. Los padres bajo este estilo de crianza imponen reglas, tanto morales como de comportamiento, las cuales deben ser practicadas sin objeción; no toleran las contradicciones y actúan aún en contra de los intereses o aspiraciones de los hijos. Justifican su actitud partiendo del principio de que ese es el camino para que los hijos tengan un futuro favorable, y que la "mano dura" hace hijos responsables y comprometidos.

De acuerdo a los hallazgos dentro de esta línea de investigación (Aguilar et al., 2004; Lambord et al., 1991; Jiménez, 2000), existen ciertas variables que suelen estar fuertemente asociadas con los estilos de crianza específicos. Entre tales variables relacionadas se pueden ubicar: 1) el nivel de escolaridad materna y paterna, dado que altos niveles de escolaridad suelen relacionarse con los estilos democrático y permisivo, mientras que en padres y madres con niveles escolares bajos pueden ubicarse los estilos autoritario y negligente, y 2) clase social, la relación es similar a la reportada para la variable escolaridad paterna y materna, aunque con menor grado de influencia. Además, existen investigaciones (Berridi, 2001; Jiménez, 2000; Steinberg et al., 1994; Vallejo, 2002) que prueban que los estilos de crianza paternos influyen fuertemente sobre tres variables específicas en sus hijos: rendimiento académico, nivel de auto-estima y conducta social dentro del ámbito escolar y familiar. El estilo democrático se relaciona con niveles satisfactorios de rendimiento académico y auto-estima, así como con conducta social adecuada en escuela y familia por parte de los niños, mientras que los otros estilos se correlacionan con bajos niveles de rendimiento escolar, de auto-estima y/o de conducta social.

Desde una perspectiva conductual, otra línea de investigación se ha encargado de estudiar el tema, enfocando las formas específicas de interac- 
ción en el hogar, especialmente las interacciones diádicas madre-hijo, así como su impacto sobre el desarrollo psicológico infantil. Esta tendencia se contrapone a la concepción tradicional y unidireccional que plantea que son las madres quienes, a través de sus estilos de crianza, determinan las características de la relación con sus hijos y por ende el desarrollo infantil; en lugar de ello, se adopta una concepción interactiva de la relación diádica, así como del proceso de desarrollo infantil y académico, donde confluyen factores como el contexto físico y social, el nivel cultural y las actividades realizadas dentro del hogar como parte de la vida interactiva cotidiana (Guevara y Mares, 1995). Este enfoque parte de que el comportamiento de los padres en situaciones de interacción con los niños depende de las características y tipo de conductas de éstos, y que a su vez, la conducta infantil varía de acuerdo a las características y conductas de los progenitores (especialmente de la madre), así como de la situación particular que se viva. Autores como Pineda (1986) plantean que cuando el niño es expuesto a un ambiente rico, donde hay muchos objetos y actividades cuyo acceso sólo es posible a través de la comunicación, se promueven mejores interacciones entre niños y adultos, lo cual repercute de manera importante en el desarrollo infantil.

Torres, Ortega, Reyes y Garrido (2008) reportan que las interacciones diádicas se pueden diferenciar de acuerdo a su calidad. La calidad de la interacción madre-niño puede verse influida por el momento y la topografía en que se den las respuestas a la otra persona, dado que la respuesta de un integrante generalmente precede a la del otro, con lo cual se da una sincronía de respuestas. Dentro de las interacciones de alta calidad, las autoras ubican aquellas en donde las madres observan y responden a las necesidades del niño, es decir son sensitivas y responsivas a las conductas presentadas por su hijo, inician y promueven situaciones interactivas, integrándose en actividades conjuntas con su hijo, sin recurrir a conductas que pudieran restringir la adquisición de nuevas habilidades por parte del niño, como la directividad o conductas autoritarias. El desarrollo de conductas mutuamente reforzantes durante los primeros años de vida del niño aumenta la probabilidad de que se mantenga la satisfacción y la cooperación mutua, y a partir de esto, surjan estilos de interacción que sean promotores de un buen desarrollo psicológico infantil.

Connell y Prinz (2002) reportan que el nivel de escolaridad de la madre se relaciona con las interacciones diádicas adecuadas y cercanas, y que dichos patrones de interacción se relacionan a su vez con niveles altos de habilidades sociales y de comunicación en los niños. Mientras que estudios longitudinales (Baker, Mackler, Sonnenschein y Serpell, 2001; Morrison, Rimm-Kauffman y Pianta, 2003) encuentran una fuerte relación entre los patrones de interacción temprana madre-hijo y los resultados académicos de los alumnos, así como sus conductas sociales, en el transcurso de su educación básica. Estos autores concluyen que en díadas con relaciones tempranas 
negativas, los niños se encuentren en riesgo de tener resultados escolares negativos. Similarmente, diferentes investigaciones (Connor, Son, Hindman, y Morrison, 2005; Hughes, Gleason y Zhang, 2005; Peñalva, 2001) indican que las actitudes de los alumnos con referencia a la escuela, al profesor y a las actividades culturales, están mediadas por el tipo de interacciones que el niño establece en el hogar, y que dichas variables se asocian con el rendimiento académico.

Dados los hallazgos de las líneas de investigación mencionadas, resulta clara la necesidad de desarrollar estrategias que permitan a las madres adquirir habilidades para acercarse a sus hijos, así como conductas que correspondan a un estilo de crianza democrático. Promover mejores interacciones diádicas madre-hijo puede ser una buena manera de mejorar el aprovechamiento escolar de los alumnos, así como su adaptación social, escolar y familiar. Dicha necesidad se presenta especialmente en familias que pueden mostrar algunos factores de riesgo como un nivel sociocultural bajo, o bien con alumnos que desde los primeros años escolares ya muestran problemas de conducta en el aula o bajo rendimiento académico. También resulta importante clarificar cuál es la mejor estrategia para lograr esos objetivos, si brindar un entrenamiento exclusivamente a las madres con el objeto de mejorar sus prácticas de crianza (tal como lo señalan diversos autores como Epstein, 2001 y Martínez, Martínez y Pérez, 2004), o involucrar a ambos miembros de la díada -madre e hijo- en un entrenamiento encaminado a mejorar sus interacciones.

El objetivo central de la presente investigación fue evaluar la efectividad de dos estrategias de intervención en las interacciones madre-hijo, su relación con el desempeño académico y social de los niños.

\section{MÉTODO}

\section{Participantes}

En el estudio se contó con 90 participantes, 45 madres y sus hijos, 18 niñas y 27 niños con una media de edad de 5.9 años, inscritos en el primer grado de una escuela primaria pública ubicada en una colonia de nivel sociocultural bajo, en la zona metropolitana del Estado de México (ver Anexo 1). El criterio para la inclusión de los niños en el estudio fue que hubieran obtenido un promedio de calificación global menor de 7 durante la primera evaluación bimestral del ciclo escolar 2006-2007 y que, junto con sus madres, estuvieran dispuestos a participar en el estudio. El $44 \%$ de los alumnos participantes fue reportado por sus profesores con conducta inadecuada dentro del salón de clases. De acuerdo con los reportes de las madres participantes, el $47 \%$ de ellas cuenta con un ingreso mensual familiar entre mil y cuatro mil pesos, el 
$87 \%$ tiene una escolaridad máxima de nivel secundaria, el $56 \%$ se dedica al hogar, el $67 \%$ están casadas, el $4 \%$ de sus parejas no tiene actividad laboral y el $96 \%$ tiene una actividad laboral fija u ocasional, el $42 \%$ tiene entre tres y cinco hijos y la edad de las madres participantes se ubicó entre 23 y 44 años, con un promedio de 32.4 años.

\section{Variables}

Las variables de la investigación correspondieron a tres categorías: demográficas, dependientes e independientes. Dentro de las primeras se ubicaron: edad y sexo del niño, edad, estado civil, escolaridad y ocupación de la madre, así como estructura e ingreso mensual familiar.

Las variables dependientes fueron: 1. Estilos de crianza reportados por las madres, definidos en términos del porcentaje de reactivos que las madres contestaron como "frecuentemente" y "muy frecuentemente", en respuesta a las preguntas realizadas acerca de la forma en que actúan ante situaciones particulares con sus hijos; tales respuestas permitieron ubicar los niveles en que se presentó cada uno de los cuatro estilos de crianza (democrático, permisivo, negligente y autoritario) que explora la "Escala sobre estilos maternos de crianza" elaborado por Jiménez (2000). 2. Estilos de crianza reportados por los hijos, definidos como el porcentaje de reactivos que los niños eligieron en respuesta a las preguntas realizadas acerca de la forma en que actúan sus madres ante situaciones particulares con ellos, que explora la "Escala de reportes del niño acerca del estilo de crianza materno" de Jiménez (2000). 3. Rendimiento académico de los alumnos participantes, que contempló los puntajes de las calificaciones bimestrales oficiales de las tres materias académicas del currículum o plan de estudios de primer grado de primaria, Matemáticas, Español y Conocimiento del medio, relacionadas con habilidades operacionales, de lecto-escritura y de conocimiento del medio ambiente; son obtenidas a través de los criterios que la Secretaría de Educación Pública determina para evaluar el avance académico de los alumnos, incluye participación en clase, cumplimiento de tareas académicas y ejecución en los exámenes bimestrales diseñados con base en los contenidos temáticos revisados durante el periodo correspondiente. 4. Conductas sociales inadecuadas en el aula, definidas (de acuerdo con Plaza, 1996), como aquellas que influyen negativamente en el proceso docente y/o suponen un trastorno para el desarrollo de la vida escolar; entre ellas se ubican: negarse a cumplir las reglas o tareas, molestar a compañeros o profesores, desobediencia sistemática y agresión física o verbal; para el presente estudio se consideró el número de niños cuyos profesores identificaron con alguna(s) de estas conductas, con base en el cuestionario utilizado para ello.

La variable independiente fue la estrategia de intervención. Se compararon dos estrategias generales: la primera fue la intervención con un grupo 
de madres y sus hijos, para mejorar sus patrones de interacción diádica; la segunda fue el entrenamiento dirigido a madres, orientado a modificar sus estilos de crianza y promover en ellas el estilo democrático con sus hijos. Ambas estrategias fueron comparadas entre sí, al comparar a los grupos que recibieron uno y otro programa, así como con el grupo control que no recibió ninguno de los programas durante el ciclo escolar referido.

\section{Instrumentos}

1. Cuestionario sobre datos demográficos elaborado por Jiménez (2000).

2. La Escala sobre estilos maternos de crianza, dirigido a madres está conformada por 80 reactivos que presentan 17 situaciones de interacción diádica. La autora reporta que se balancearon tanto los reactivos como las situaciones para mantener el equilibrio de la prueba; las respuestas a cada situación hipotética pretenden abarcar alguna característica de interacción de los diferentes estilos de crianza. Las situaciones contemplan diferentes dimensiones de interacción madre-hijo: a) acciones pro-sociales, labores del hogar y conductas de ayuda; b) demandas para conductas apropiadas y limitación de conductas inapropiadas; c) demandas de cuidado, que incluyen auto-cuidado y regulación de seguridad física y de orientación del niño, y d) cómo las madres hacen peticiones a sus hijos. Delante de cada reactivo se ubican cuatro opciones de respuesta, de acuerdo a lo que la madre cotidianamente haría en dicha situación, ellas deben elegir lo que hacen: Muy frecuentemente, Frecuentemente, Casi nunca y Nunca (hay 20 reactivos para ubicar cada estilo de crianza). Los datos de diseño, validez y confiabilidad fueron reportados por su autora (Jiménez, 2000).

3. La Escala de reportes del niño acerca del estilo de crianza materno contiene 32 reactivos planteados gráficamente y en forma de situaciones cotidianas para el niño, las cuales se dividen en tres categorías o escenarios: hogar, escuela y social. Cada reactivo cuenta con cuatro opciones de respuesta, donde cada una de ellas corresponde a uno de los estilos de crianza referidos. Los dos primeros reactivos tienen como objetivo que el niño entendiera el funcionamiento del cuestionario, por lo que se les llama "reactivos de entrenamiento", no se evalúan. Al igual que el instrumento anterior, la prueba fue diseñada, validada y confiabilizada por su autora (Jiménez, 2000).

4. El cuestionario dirigido a los profesores de primer grado se conformó con preguntas relacionadas con la conducta de cada uno de sus alumnos dentro del salón de clase: si el alumno cumplía con las reglas establecidas dentro del salón de clases, si las tareas asignadas eran cumplidas satisfactoriamente, si la conducta del alumno se orientaba a molestar a compañeros o profesores, si existía desobediencia sistemática en el 
horario de clases, si consideraba que el alumno manifestaba algún tipo de agresión física o verbal hacia los demás. Lo anterior se consideró con base en la literatura acerca del tema (Plaza, 1996). Criterio de evaluación del cuestionario: se consideraba que un alumno presentaba conducta inadecuada en el aula, aun cuando el profesor ubicara en él una sola de las conductas problema.

5. Las calificaciones oficiales. Se consideraron únicamente las calificaciones de las materias académicas básicas -Español, Matemáticas y Conocimiento del medio-, para que no influyeran las calificaciones asignadas por el profesor para aspectos como puntualidad, disciplina, aseo 0 cuidado personal, actividades artísticas o actividades deportivas.

Obtención y análisis de los datos

Las variables demográficas fueron consideradas únicamente como descriptoras. Las medidas de las variables dependientes se obtuvieron de la manera siguiente: 1) para ubicar las conductas maternas predominantes durante la interacción con sus hijos, se consideraron sólo aquellas reactivos que las propias madres contestaron con las opciones "frecuentemente" y "muy frecuentemente", catalogadas dentro de cada uno de los cuatro estilos de crianza; se obtuvo el porcentaje de las conductas de cada estilo sumando dichas respuestas y dividiendo entre el total de reactivos; 2) las respuestas dadas por los niños acerca de las conductas maternas fueron sumadas por estilo y divididas entre el total de reactivos, para obtener su porcentaje; 3) se consideró el número de alumnos reportados con conducta inadecuada en el aula; 4) se obtuvo el promedio de las calificaciones académicas para cada materia y para la calificación académica global, a lo largo del ciclo escolar.

Con los porcentajes y promedios mencionados se elaboraron gráficas que permiten comparar el desempeño de las madres y los alumnos de cada grupo, antes y después de la intervención. Para contar con datos adicionales se realizaron pruebas estadísticas utilizando el programa SPSS versión 12.0 para Windows.

\section{Procedimiento}

El estudio tuvo un diseño pretest-postest, con dos grupos experimentales y un grupo control (siguiendo los criterios de Méndez, Namihira, Moreno y Sosa, 2006).

Fase de pre-evaluación

Después de explicarles los propósitos del estudio, las madres interesadas firmaron un consentimiento informado sobre su participación y la de sus hijos. Los investigadores aplicaron los instrumentos. El cuestionario sobre datos demográficos y la escala sobre estilos maternos de crianza, dirigidos a las 
madres, fueron contestados por escrito en una sesión grupal de 50 minutos. Para los alumnos, la aplicación del instrumento se realizó de manera individual en sesiones de 35 minutos, aproximadamente. La aplicación del cuestionario dirigido a profesores para informar problemas de conducta en el aula tuvo una duración de 10 minutos para el reporte de cada niño.

Una vez concluida la fase de pre-evaluación, las 45 díadas fueron asignadas de manera aleatoria a tres distintas situaciones experimentales, conformándose dos grupos de intervención y uno control, con 15 díadas cada uno. Para asegurar que antes de empezar el entrenamiento no hubiera diferencias importantes entre los grupos, se realizó el análisis estadístico (chi cuadrada y ANOVA), el cual indicó que no hubo diferencias estadísticamente significativas entre ellos, ni en lo relativo a las variables demográficas ni en promedio escolar y conducta inadecuada de los alumnos.

Fase de intervención

La aplicación de las dos estrategias de intervención se llevó a cabo por parte del mismo investigador, en el mismo escenario (un aula de la escuela, de aproximadamente $8 \times 10$ metros, equipada con dos pizarrones y 30 mesabancos), y de manera paralela, en diferentes días de la semana.

Grupo A. Entrenamiento con díadas. Las 15 díadas de este grupo recibieron conjuntamente una estrategia de entrenamiento sobre formas específicas de interacción entre ambos miembros de la díada; el programa se aplicó durante ocho sesiones grupales de aproximadamente dos horas de duración, con un intervalo entre sesiones de una semana. Posteriormente cada una de las díadas recibió cuatro sesiones adicionales.

Cada una de las sesiones grupales inició con el planteamiento de un objetivo específico por parte del investigador, objetivo que fue escrito en el pizarrón. La primera hora de cada sesión grupal fue llevada a cabo exclusivamente con las madres, sin que estuvieran los hijos presentes. Esta parte del entrenamiento se encaminó a desarrollar en las madres habilidades para establecer reglas y rutinas dentro y fuera del hogar, acuerdos de convivencia y participación en actividades familiares, apoyar las actividades escolares y reconocer los logros de los niños; todo ello promoviendo que los niños participen y sin que las madres utilicen golpes o insultos.

En estas sesiones, el investigador expuso a las madres de manera sencilla una serie de planteamientos de la psicología -algunos aspectos teóricos acerca de la influencia de la familia en el desarrollo de los niños y algunas técnicas que se utilizan para la educación infantil, tales como la retroalimentación positiva, el reforzamiento y la asertividad-, encaminados a propiciar interacciones positivas y negociaciones entre las díadas. También se proporcionó modelamiento de dichas negociaciones e interacciones, a través de un juego de roles, donde el instructor fungió como padre y el asistente como 
su hijo. Después se discutía en el grupo de madres acerca de las ventajas de dichas estrategias del estilo democrático y de las desventajas de utilizar métodos autoritarios con sus hijos.

Durante la segunda hora de cada sesión grupal se incorporaban los niños, entonces se procedió a realizar un nuevo juego de roles, en donde el investigador y su asistente modelaban una interacción positiva o negociación diádica, y después actuaban dos o tres díadas madre-hijo para practicar estas formas de interacción positiva y democrática. Al final de cada sesión, todos los participantes retroalimentaban a las díadas que actuaban y analizaban errores y aciertos de cada díada, tomando en consideración el modelo, las instrucciones y el objetivo de la sesión. Se cuidó que todas las díadas participaran. De esta manera se trabajaron las siguientes actividades en las ocho sesiones grupales: 1) establecimiento de reglas y rutinas en el hogar, incluyendo horarios para levantarse, desayunar, arreglarse, hacer la tarea, ver televisión, jugar y dormir; 2) formas de interacción, incluyendo peticiones de madre e hijo, respuestas a peticiones y discusión adecuada de desacuerdos; 3) negociación para actividades fuera de casa, tales como visitas a familiares y asistencia a eventos sociales; 4) establecer las consecuencias de romper acuerdos o reglas establecidas por la díada; 5) dirigirse al otro de manera afectiva y comprensiva en situaciones de conflicto; 6 ) trasmitirle confianza al otro; 7) apoyo y reconocimiento de las actividades escolares de los niños, y 8) apoyo y reconocimiento de las actividades maternas.

En las cuatro sesiones finales, donde el investigador trabajó con cada díada por separado, se abordaron aspectos específicos del programa grupal que la madre o el hijo tuvieran interés en retomar, ya fuera lo relativo a un tema de los ya tratados o a la manera de solucionar un conflicto específico entre ellos.

Grupo B. Entrenamiento con madres. Las 15 madres de este grupo recibieron entrenamiento mediante una estrategia orientada a desarrollar habilidades de interacción y de negociación con sus hijos y a establecer acuerdos con la participación de ambos miembros de la díada, es decir, se intentó modificar la tendencia que las madres pudieran tener hacia un estilo de crianza en particular, a través de promover en ellas conductas relacionadas con el estilo de crianza democrático. El entrenamiento se llevó a cabo a lo largo de doce sesiones grupales de aproximadamente dos horas de duración, con un intervalo entre sesiones de una semana. El procedimiento general de esta estrategia, las técnicas de entrenamiento, los temas tratados y las actividades del programa, fueron los mismos que en la estrategia anterior, la única diferencia fue que no participaron los niños. Se entrenaron en las madres habilidades de negociación y establecimiento de reglas en actividades cotidianas, haciendo partícipe a su hijo en la toma de decisiones, considerando su punto de vista y procurando llegar a acuerdos mutuos. 
Cada una de las doce sesiones grupales inició con el planteamiento, por parte del investigador, de un objetivo específico que se escribió en el pizarrón. Al igual que en el programa anterior, el investigador expuso a las madres de manera sencilla una serie de planteamientos de la psicología -algunos aspectos teóricos acerca de la influencia de la familia en el desarrollo de los niños y algunas técnicas que se utilizan para la educación infantil, tales como la retroalimentación positiva, el reforzamiento y la asertividad-, encaminados para promover conductas que se ubican dentro del estilo de crianza democrático. Mediante el modelamiento y un juego de roles, el investigador fungió como padre y el asistente como su hijo. Después se discutió con el grupo de madres, acerca de las ventajas de dichas estrategias democráticas y de las desventajas de utilizar métodos autoritarios o de ser negligentes con los hijos. Posteriormente se procedió a realizar un nuevo juego de roles, en donde el investigador o su asistente fungían como hijo, para que cada madre practicara las formas de interacción diádica en actividades cotidianas, en cada ejemplo expuesto. Al final de cada sesión, el investigador y el grupo de madres retroalimentaban a las participantes del juego de roles, analizando sus errores y aciertos, tomando en consideración el modelo, las instrucciones y el objetivo de la sesión. Se cuidó que todas las madres participaran en el juego de roles y en la retroalimentación.

Grupo C. Control. Las 15 díadas que fungieron como grupo control no recibieron ninguno de los dos entrenamientos. Por razones éticas se programó el entrenamiento a este grupo de manera diferida, una vez concluido el estudio.

Fase de pos-evaluación

Se llevó a cabo la segunda aplicación de los instrumentos: ambas escalas de estilo de crianza materno, a los 45 niños y a las 45 madres participantes, de la misma manera que en pre-evaluación; a los profesores se les pidió nuevamente que llenaran el cuestionario relacionado con la conducta inadecuada dentro del salón de clase de cada uno de sus alumnos, cabe mencionar que los profesores no conocían en qué situación experimental se encontraba cada alumno. Además de lo anterior, a lo largo del ciclo escolar se solicitó al director de la escuela primaria que proporcionara los puntajes de las calificaciones obtenidas por los alumnos de primer grado en cada bimestre, de las tres materias académicas.

\section{RESULTADOS}

Descripción general del desempeño de los grupos durante el entrenamiento En ambos grupos de entrenamiento las madres participaron activamente a lo largo de las sesiones, realizando preguntas específicas de acuerdo a las 
experiencias cotidianas con sus hijos. Durante el juego de roles, cooperaban entre ellas para que se dieran interacciones positivas y mostraban interés en la ejecución de todas; durante las sesiones iniciales se requirió frecuentemente la participación del investigador para proporcionar retroalimentación, modelamiento o instrucciones adicionales a algunas díadas durante las representaciones, lo cual fue disminuyendo conforme avanzaba el entrenamiento, para las sesiones finales las interacciones diádicas positivas fueron muy frecuentes en todas las participantes.

En el caso del Grupo A se observó además que, cuando los niños participaban en el juego de roles, las madres solían encaminarlos para interactuar adecuadamente. Al final de las sesiones, cuando el grupo retroalimentaba a las díadas participantes, tanto madres como hijos expresaban su opinión acerca de las interacciones representadas. Este espacio también sirvió para que cada díada estableciera acuerdos y los asentaran por escrito. En las cuatro sesiones finales, donde el investigador atendió a cada díada por separado, las actividades estuvieron dirigidas principalmente a las negociaciones entre madre e hijo.

\section{Estilos de crianza reportados por madres e hijos}

La Figura 1 muestra la comparación de los porcentajes obtenidos en las respuestas correspondientes a cada uno de los estilos de crianza, de acuerdo a lo reportado por las madres de los tres grupos de investigación, antes y después de la aplicación de los programas de intervención. Durante la evaluación inicial no se observaron diferencias entre los reportes de las madres de los tres grupos. En todos los casos, se ubicaron porcentajes menores al $25 \%$ para respuestas correspondientes a los estilos de crianza catalogados como negligente y permisivo. Las respuestas predominantes correspondieron a los estilos democrático y autoritario, con poca diferencia entre sus respectivos porcentajes. Para la evaluación final, los reportes indicaron modificaciones en la tendencia de las madres a responder con dichos patrones y reflejaron diferencias entre los tres grupos de madres. En el Grupo A, las madres reportaron un incremento de las respuestas correspondientes al estilo democrático, alcanzando un nivel del $47 \%$, así como un decremento en las que se ubican dentro del estilo negligente, alcanzando un nivel cercano a $0 \%$, en tanto que las respuestas propias de los estilos permisivo y autoritario no mostraron cambio. En el Grupo B, las madres reportaron un incremento de las respuestas ubicadas dentro del estilo democrático, similar al del Grupo A, así como una permanencia de los niveles de respuesta ubicadas en los estilos negligente y permisivo; en este caso, las respuestas que bajaron su porcentaje fueron las correspondientes al estilo autoritario. En el Grupo C no se presentó ningún cambio en los reportes de las madres. 

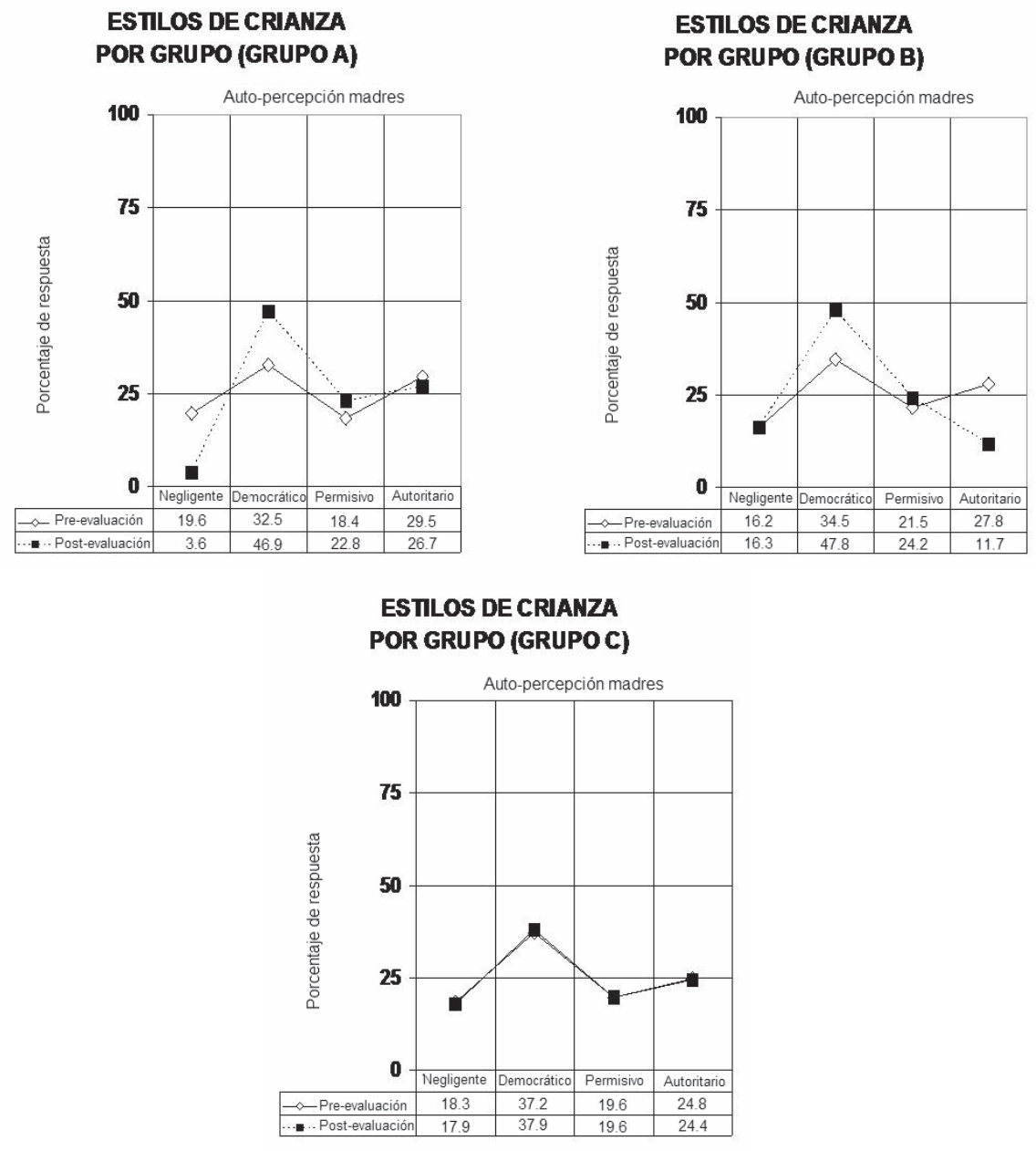

Figura 1. Comparación de los porcentajes promedio de respuestas reportadas por las madres de cada grupo, correspondientes a cada estilo de crianza, en pre-evaluación y pos-evaluación. Nota: en el caso del Grupo C, los datos de pre-evaluación y pos-evaluación fueron los mismos, por ello no se aprecian los primeros.

La Figura 2 muestra los resultados de los reportes infantiles acerca de las tendencias de sus madres a comportarse dentro de cada uno de los estilos de crianza; se comparan los porcentajes obtenidos por los tres grupos de 
investigación, antes y después de la aplicación de los programas de intervención. Durante la evaluación inicial no se observaron diferencias entre los porcentajes que reportaron los niños de los tres grupos. En todos los casos, se ubicaron bajos niveles de conductas maternas correspondientes al estilo de crianza catalogado como negligente, entre 0 y 4 de los 30 reactivos de la prueba fueron contestados con la opción que corresponde a este estilo, por lo que su porcentaje máximo fue del $14 \%$; en segundo lugar se ubicaron las respuestas maternas propias de los estilos democrático y permisivo, entre 6 y 9 de los reactivos fueron contestados con las opciones que corresponden a éstos, por lo que sus porcentajes estuvieron entre 20 y $30 \%$ en ambos casos; las conductas maternas que caen en el estilo autoritario fueron las de mayor porcentaje, entre 10 y 16 de las 30 respuestas al instrumento correspondieron a este estilo materno, lo que representa porcentajes entre 35 y $56 \%$. Después de la intervención, se observaron diferencias entre los grupos. Los niños del Grupo A disminuyeron el porcentaje de reportes sobre las conductas maternas que se ubican en los estilos negligente, permisivo y autoritario, aumentando notablemente los reportes de conductas maternas que caen en el estilo democrático; con un promedio cercano a 20 respuestas (65\%), las conductas maternas relacionadas con el estilo democrático se convirtieron en las predominantes para este grupo. Los niños de los grupos $\mathrm{B}$ y $\mathrm{C}$ reportaron porcentajes de conductas maternas muy similares a los reportados durante la evaluación inicial, con predominancia de las conductas que pueden ubicarse en el estilo autoritario. 

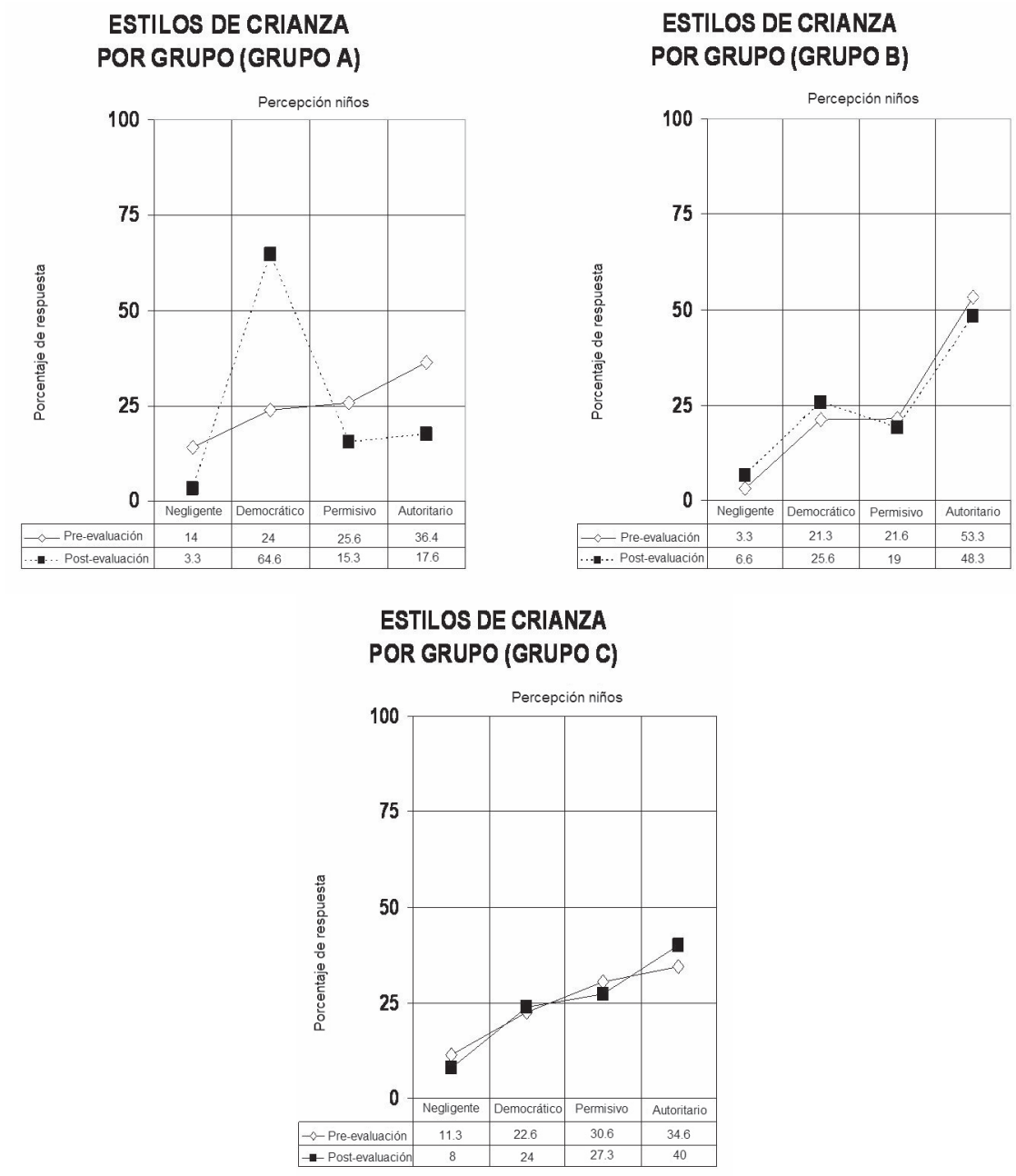

Figura 2. Comparación de los porcentajes promedio por grupo, correspondientes a las conductas que los niños reportan en sus madres, ubicadas dentro de cada estilo de crianza, en pre-evaluación y pos-evaluación.

Conducta en el aula y desempeño académico

De acuerdo con el reporte de los profesores, durante la evaluación inicial 18 de los 45 niños participantes se ubicaron como alumnos con conducta inadecuada, de los cuales 33\% formaron parte del Grupo A, 38\% del Grupo B y 
$28 \%$ del Grupo Control. Para contar con un dato adicional, se realizó un análisis de chi cuadrada, que indicó que no existieron diferencias significativas en la conducta inadecuada entre los alumnos de los tres grupos. Al final del ciclo escolar, el número de niños con conducta inadecuada se redujo a 14, de los cuales sólo uno formaba parte del Grupo A, ocho del Grupo B y cinco del Grupo C. En esta ocasión, la chi cuadrada indicó que tales diferencias entre los grupos fueron estadísticamente significativas en la post-evaluación $(p=.022)$.

Para medir el impacto de los programas de entrenamiento sobre el desempeño académico de los alumnos se consideraron las calificaciones de las tres materias académicas del curso -Matemáticas, Español y Conocimiento del medio-, obtenidas por los niños en cada uno de los cinco bimestres que comprenden el ciclo escolar. Con tales datos se obtuvo el promedio de desempeño académico de cada uno de los grupos participantes, por bimestre, para cada una de las tres materias. También se obtuvo una calificación académica global promediando las calificaciones de estas tres materias. En la Figura 3 se muestran los avances en el desempeño académico de los alumnos de los tres grupos del estudio. En este caso, también se obtuvieron datos adicionales realizando comparaciones estadísticas de las calificaciones de los grupos a través de la aplicación de un análisis de varianza (ANOVA), así como de una prueba $t$ de Student para muestras relacionadas que permitió conocer el grado de avance académico de cada grupo. 
MATEMATICAS

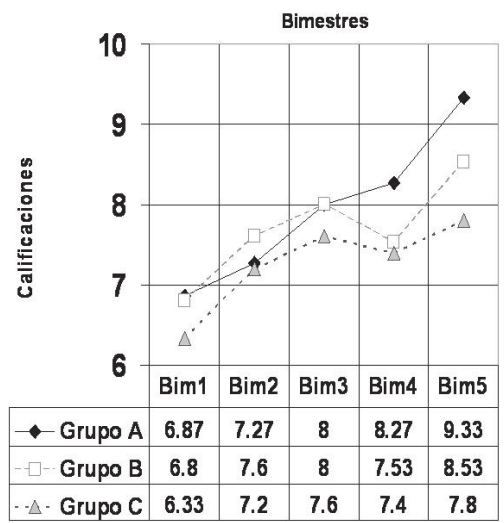

Registro de las calificaciones de la materia de Matemáticas de cada grupo del ańo escolar 2006-2007

\section{CONOCIMIENTO DEL MEDIO}

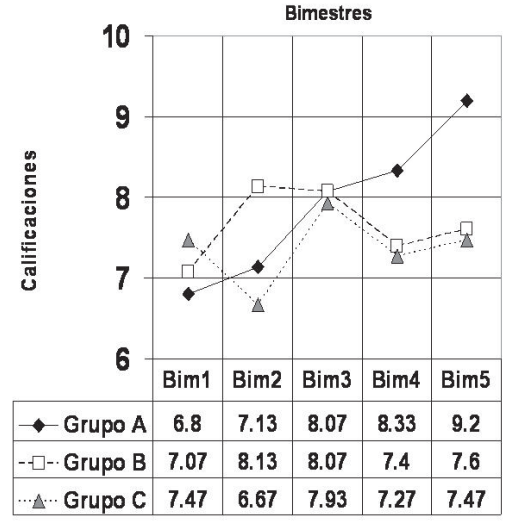

Registro de las calificaciones de la materia de Conocimiento del medio de cada grupo del año escolar 2006-2007
ESPAÑOL

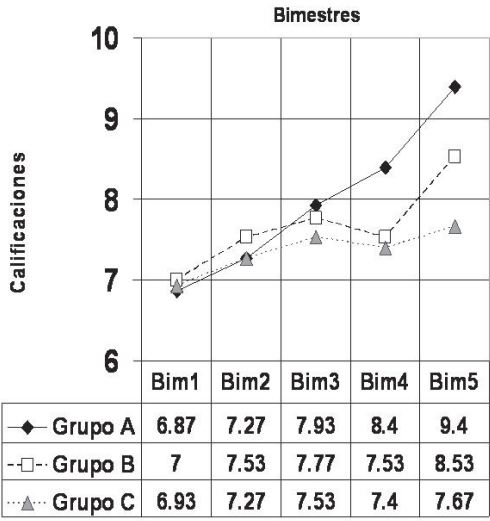

Registro de las calificaciones de la materia de Español de cada grupo del año escolar 2006-2007

PROMEDIO GLOBAL

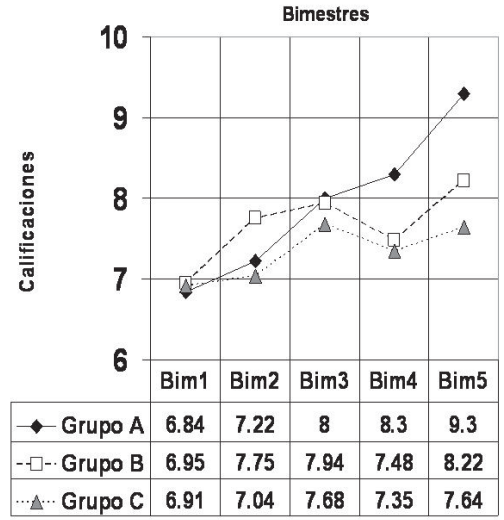

Registro del promedio bimestral de las tres materias básicas (Matemáticas, Español, Conocimiento del medio)

Figura 3. Promedio bimestral del desempeño de los alumnos en las tres materias académicas básicas (Matemáticas, Español, Conocimiento del medio) y su promedio académico global, por grupo. 
Como se aprecia en la Figura 3, durante el primer bimestre del ciclo escolar (Fase de pre-evaluación del presente estudio), los promedios académicos de los alumnos fueron muy similares para los tres grupos, por debajo de 7 de calificación global (dado que ese fue uno de los criterios de selección de los participantes); esta similitud en el desempeño académico de los alumnos se observó también en el promedio de cada una de las tres materias consideradas. El dato proporcionado por el ANOVA indicó que en este momento del ciclo escolar no hubo diferencias estadísticamente significativas entre los grupos, en lo relativo a las materias Español y Conocimiento del medio, ni en la calificación académica global, aunque los dos grupos de entrenamiento (A y B) tuvieron calificaciones ligeramente superiores que el Grupo Control en la materia Matemáticas $(p=.041)$.

Para el segundo bimestre, es decir, al finalizar la cuarta sesión de los programas de entrenamiento aplicados a los dos grupos experimentales, se observó que casi todos los promedios de calificación de los alumnos mostraron incrementos (la prueba $t$ indicó que dichos incrementos no fueron estadísticamente significativos). En este momento del estudio, fueron mayores los avances del Grupo B, aunque al realizar el ANOVA se encontró que, en lo relativo a Matemáticas, Español y en la calificación académica global, las diferencias entre grupos no fueron estadísticamente significativas.

Para el tercer bimestre se habían cubierto ya ocho sesiones de los programas de entrenamiento, en la Figura 3 se aprecia que hubo nuevamente un incremento en las calificaciones de las tres materias, en los tres grupos. Esta vez las calificaciones académicas de los alumnos del Grupo A fueron ligeramente superiores, alcanzando promedios de alrededor de 8 de calificación, aunque las diferencias entre grupos no fueron estadísticamente significativas.

El cuarto bimestre correspondió en tiempo con la sesión 12 de los programas de entrenamiento; aquí se pudo apreciar que los promedios académicos de los grupos B y C bajaron ligeramente, para ubicarse por debajo de 8 en todos los casos, en tanto que los promedios académicos de los alumnos del Grupo A continuaron aumentando. Al obtener los datos del ANOVA, éste arrojó diferencias estadísticamente significativas a favor del Grupo A, en las tres materias consideradas en el análisis, así como en el promedio académico global (Matemáticas, $p=.009$; Español, $p=.001$; Conocimiento del medio y calificación global, $p=.000$ ).

Cuando los profesores asentaron en las boletas las calificaciones académicas del quinto bimestre, los programas de entrenamiento tenían dos meses de haber concluido. Lo que pudo observarse en ese momento fue que todos los promedios de calificación mostraron nuevos incrementos, mínimos para el caso del Grupo C (que llegó a 7.6 de calificación académica global), un poco mayores en el Grupo B (que alcanzó 8.2 de calificación académica 
global), y en el Grupo A se alcanzaron promedios mayores a 9 de calificación en todos los casos. La prueba $t$ se llevó a cabo en cada grupo, para comparar los promedio académicos iniciales con los finales, se encontró que los alumnos de los tres grupos mejoraron su ejecución de manera significativa $(p=.000)$, excepto en el caso de la materia Conocimiento del medio para los grupos C y B. Por su parte, el ANOVA indicó que las diferencias entre grupos fueron estadísticamente significativas $(p=.000)$, a favor del Grupo A, en las tres materias consideradas y en la calificación académica global.

\section{DISCUSIÓN Y CONCLUSIONES}

Los hallazgos de la evaluación inicial de esta investigación confirman lo reportado por diversos autores (Aguilar et al., 2004; Connell y Prinz, 2002; Jiménez, 2000; Lambord et al., 1991), en el sentido de que las madres de nivel sociocultural bajo muestran una tendencia hacia el estilo autoritario. Antes de la intervención, las madres reportaron como conductas predominantes las relacionadas con ese estilo de crianza, aunque ligeramente por debajo de las conductas ubicadas en el estilo democrático. Dicha tendencia materna fue aún más evidente cuando se consideraron los reportes de los niños de los tres grupos participantes, quienes calificaron las conductas de sus madres con una franca predominancia hacia el estilo autoritario, y con algunos indicios conductuales de los estilos democrático y permisivo. De hecho, en ese momento de la investigación se pudo observar que, si bien no existió una plena correspondencia entre los reportes maternos y los reportes de sus hijos, sí hubo algunas coincidencias; además de las antes mencionadas, en todos los casos las conductas maternas que se pueden ubicar en el estilo negligente fueron las de menor porcentaje.

Los datos de la evaluación realizada después de la intervención indican que el programa de entrenamiento impartido a los participantes del Grupo A tuvo como efecto la modificación de sus patrones de interacción diádica. En esa segunda aplicación del instrumento, los reportes de los niños del Grupo A indicaron una predominancia de sus madres a mostrar conductas que se pueden ubicar dentro del estilo de crianza democrático, con disminución de los porcentajes de conducta correspondientes a los otros tres estilos. En este grupo se observó coincidencia entre lo reportado por las madres y lo reportado por sus hijos respecto a los tipos de conducta materna exhibida.

En el caso de los participantes del Grupo B se observó un cambio, según las madres sus niveles de conductas ubicadas en el estilo autoritario bajaron aún más, y sus conductas correspondientes al estilo democrático aumentaron en la misma proporción, incrementándose por tanto su nivel de predominancia. Sin embargo, esos reportes maternos no coincidieron con 
los de sus hijos, dado que los alumnos de este grupo siguieron ubicando las conductas correspondientes al estilo autoritario en sus madres, como las de mayor presencia. Estos hallazgos nos hacen suponer que las madres respondieron en las evaluaciones tratando de ajustarse a lo que ellas juzgaron socialmente deseable, especialmente para el caso de la pos-evaluación; dado que durante el entrenamiento tanto el investigador como el grupo de madres se centraron en impulsar entre ellas las conductas que se ajustan al estilo democrático, así como a disminuir las otras, las respuestas dadas a los reactivos pueden haber reflejado lo que ellas sabían que debían contestar, no necesariamente las conductas que ejercen con sus hijos. Desde luego, también puede haberse dado el caso de que las madres de este Grupo B realmente hubieran modificado sus conductas, encaminándolas a un estilo democrático, pero que sus hijos aún no percibieran plenamente dicho cambio. Por su parte, ni las madres ni los alumnos del Grupo $\mathrm{C}$ mostraron cambio en los reportes de conducta materna, por lo que sus tendencias conductuales hacia cada estilo de crianza no parecen haberse alterado.

Los efectos más claros del entrenamiento se observaron en el Grupo A, cuyos integrantes se incorporaron en díadas para mejorar sus estilos interactivos. Durante el entrenamiento pudo observarse que cada uno de los miembros de la díada asumió un compromiso, y juntos aplicaron las estrategias de negociación e interacción. Desde luego, también en este caso podría suponerse que, para la aplicación final del instrumento, madres e hijos hubieran contestado de acuerdo a lo socialmente deseable, por la información que tenían después del curso. Sin embargo, los datos de cambio conductual en el aula, así como los avances académicos de los alumnos de este Grupo A indicaron que el programa de entrenamiento diádico sí tuvo un impacto sobre sus integrantes.

Al inicio de la investigación, las diferencias entre los grupos en cuanto al comportamiento inadecuado de los alumnos no fueron significativas, pero sí lo fueron al final del ciclo escolar. Las conductas inadecuadas de los alumnos del Grupo A prácticamente desaparecieron, de acuerdo al reporte de sus propios profesores, lo que no ocurrió en los niños de los otros dos grupos. Cabe recordar que los profesores no sabían en cuál de las condiciones experimentales del estudio se encontraba ubicado cada alumno.

Respecto al rendimiento académico pudo observarse un avance en los alumnos de los dos grupos de entrenamiento, prácticamente desde el segundo bimestre escolar, sin embargo el rendimiento académico de los alumnos del Grupo A continuó mejorando, lo que no ocurrió de manera tan clara en el Grupo B. Para el final del ciclo pudo observarse que: 1) los promedios de calificación de los alumnos del Grupo C -que no recibieron ninguno de los entrenamientos- se ubicaron por debajo de los promedios de los dos grupos que sí recibieron alguno de ellos; 2) el Grupo B obtuvo mejores promedios 
que cuando inició, y llegó a un nivel académico que puede considerarse bueno, y 3) el Grupo A mostró los mayores avances académicos, ubicándose a un nivel ligeramente superior al 9 de calificación en las tres materias académicas básicas y en el promedio global de éstas.

Tomando en consideración todos los indicadores utilizados en el presente estudio, puede concluirse que el programa de entrenamiento llevado a cabo únicamente con las madres de familia, para encaminar sus conductas hacia el estilo de crianza democrático, tuvo efectos sobre las actitudes de las madres participantes hacia la crianza infantil y posiblemente permitió mejorar las interacciones diádicas con sus hijos; que dicho cambio conductual materno pudo tener algún efecto sobre el rendimiento escolar de sus hijos, aunque no sobre su conducta en el aula, y tampoco sobre la manera en que los niños ubican las conductas maternas.

Por su parte, el entrenamiento llevado a cabo para mejorar los patrones de interacción entre madres e hijos, a través de la participación de las díadas (primero de manera grupal y después con atención especial a cada díada), tuvo un impacto sobre las tendencias conductuales maternas, así como sobre la manera en que sus hijos califican dichas conductas, ubicando ambos una predominancia de conductas maternas que corresponden al estilo democrático. Al parecer, se logró mejorar las formas de negociación e interacción en las díadas, y este cambio diádico tuvo un efecto importante sobre la conducta de los alumnos en el aula, así como sobre su rendimiento académico.

Los hallazgos de la presente investigación parecen indicar que un programa dirigido a madres de familia de estrato sociocultural bajo puede ayudar a mejorar las relaciones diádicas y a disminuir un latente peligro de fracaso escolar, tal como lo sugieren autores como Epstein (2001) y Martínez et al. (2004). Sin embargo, los datos apuntan a la consideración de que la mejor estrategia es el entrenamiento diádico, ya que tiene efectos sobre las interacciones madre-hijo, sobre la conducta en el aula y sobre el rendimiento académico, en mayor medida que la estrategia de entrenamiento a madres. Estos datos confirman la importancia de los planteamientos de autores como Baker et al. (2001), Connell y Prinz (2002), Guevara y Mares (1995), Pineda (1986), Torres et al. (2008) y Morrison et al. (2003), quienes asumen una concepción interactiva de la relación familiar, de las relaciones madre-hijo, así como del proceso de desarrollo infantil psicológico y académico.

La principal aportación de la presente investigación es que sus hallazgos apuntan hacia la consideración de la díada madre-hijo cuando se intente resolver un problema de rendimiento académico o de conducta del alumno en el aula, y no exclusivamente al entrenamiento a madres de familia. Esto es importante porque, cuando se intenta mejorar dichos aspectos, normalmente se piensa en que las madres deben asumir una actitud directiva hacia sus hijos "con problemas", en que son ellas quienes tienen la responsabilidad de 
"educar bien" a sus hijos, y pocas veces se enfoca la solución hacia la mejor manera de lograr que madres e hijos participen activamente en la definición de sus formas específicas de interactuar y de resolver el problema académico o conductual que se manifiesta en la escuela. Enfocar la solución de esta manera puede tener muchas ventajas, especialmente con poblaciones de nivel sociocultural bajo que, como se ha demostrado repetidamente en las investigaciones del campo, pueden mostrar una tendencia hacia la directividad y el autoritarismo, así como un riesgo de fracaso escolar o de bajo rendimiento académico en sus niños (INEE, 2004, 2006).

Sin embargo, debe reconocerse que realizar este tipo de intervenciones puede tener limitaciones. Una de ellas es que el entrenamiento diádico puede requerir tiempo y esfuerzo de los profesionales, así como de los miembros de la familia, de los cuales no siempre se dispone en la vida cotidiana dentro de las escuelas públicas mexicanas; para solventar en parte esta dificultad, una alternativa puede ser llevar a cabo convenios institucionales entre las escuelas primarias de la SEP y las instituciones de educación superior, para incorporar estudiantes de psicología que realicen esa importante labor de enlace entre escuela y familia. Una segunda limitación tiene que ver con el propio nivel educativo de las madres de familia, si éste es demasiado bajo (llegando al analfabetismo) la puesta en práctica de cualquier programa de intervención puede requerir ajustes, y el apoyo académico hacia sus hijos se verá también limitado.

Por esas razones, entre otras importantes, se hace necesario que en investigaciones posteriores se elaboren y prueben programas similares para profesores y alumnos de educación básica, encaminados a mejorar sus formas de interacción en el aula, ya que éste es otro de los aspectos que pueden poner en riesgo el cumplimiento de los objetivos educativos.

\section{REFERENCIAS}

Aguilar, J., Valencia, A. Martínez, M., Romero, P. y Lemus, L. (2004). Estilos parentales y medidas de desarrollo psicosocial en estudiantes universitarios. Revista Latina de Pensamiento y Lenguaje, 12 (1), 69-81.

Baker, L., Mackler, K., Sonnenschein, S., y Serpell, R. (2001). Parent's interactions with their first-grade children during storybook reading and relation with subsequent home reading activity and reading achievement. Journal of School Psychology, 39 (5) 415-438.

Berridi, R. (2001). Relaciones parentales, orientación al logro y desempeño escolar en niños de primaria. Tesis de Maestría. México: Universidad Nacional Autónoma de México. 
Bijou, S. W., y Baer, D. M. (1982). Psicología del desarrollo infantil. La etapa básica de la niñez temprana. México: Trillas.

Connell, C. M. y Prinz, R. J. (2002). The impact of childcare and parent-child interactions on school readiness and social skills development for low-income african children. Journal of school Psychology, 40 (2) 117-193.

Connor, C., Son, S., Hindman, A. H. \& Morrison, F. J. (2005). Teacher qualifications, classroom practices, family characteristics, and preschool experience: Complex effects on first grader's vocabulary an early reading outcomes. Journal of School Psychology, 43 (4) 343-375.

Epstein, J.L. (2001). School, family and community partnership. Preparing educators and improving schools. Boulder Colorado: Westview Press.

Guevara, Y. y Mares, G. (1995). Estudios sobre interacciones madre-hijo retardado. Revista Latina de Pensamiento y Lenguaje, 3, 101-118.

Hughes, N., Gleason, K., y Zhang, D. (2005). Relationship influences on teacher's perceptions of academic competence in academically at-risk minority and majority first grade students. Journal of School Psychology, 43 (4) 303-320.

Instituto Nacional para la Evaluación de la Educación (INEE, 2004, 2006). Resultados de las pruebas nacionales de aprovechamiento en lectura y matemáticas aplicadas al final de los ciclos escolares 2002-2003 y 2004-2005. México: Dirección de Pruebas y Medición.

Jiménez, M. (2000). Estilos de crianza materna informados por madres e hijos y su relación con el estatus sociocognitivo del niño preescolar. Tesis de Maestría. México: Universidad Nacional Autónoma de México.

Jiménez, D. y Guevara, Y. (2008). Estilos de crianza y su relación con el rendimiento escolar. En C. Mondragón, C. Avendaño, C. Olivier y J. Guerrero (Eds.) Saberes de la Psicología (Volumen I). México: FES Iztacala. UNAM. Capítulo 2, pp. 33-54.

Kantor, J. R. (1980). Psicología interconductual. Un ejemplo de construcción científica sistémica. México: Trillas.

Lambord, S., Mounts, N., Steinberg, L. \& Dornbusch, S. (1991). Patterns of competence and adjustament among adolescents from authoritative, authoritarian, indulgent, and neglectful families. Child Development, 62, 1049-1065.

Lipsitt, L.P. y Reese, H.W. (1983). Desarrollo infantil. México: Trillas.

Martínez, R.A., Martínez, R. y Pérez, M.H. (2004). Children's school assessment: Implications for family-school partnerships. International Journal of Educational Research, 41 (1) 24-39.

McKinney, P. (1981). Psicología del desarrollo. México: Manual Moderno.

Méndez, I., Namihira, D., Moreno, L. y Sosa, C. (2006). El protocolo de investigación. Lineamiento para su elaboración y análisis. México: Trillas.

Morrison, E.F. Rimm-Kauffman, S. y Pianta, R.C. (2003). A longitudinal study of mother-child interactions at school entry and social and academic outcomes in middle school. Journal of School Psychology, 41 (3), 185-200.

Ortega, P. (1994). La naturaleza de los vínculos adulto-niño con retardo en el desarrollo, desde una aproximación interaccional. Tesis de Maestría. México: Universidad Nacional Autónoma de México. 
Peñalva, M. C. (2001). Familias y bajo rendimiento escolar: estudio descriptivo. Tesis inédita de Doctorado. México: Universidad Nacional Autónoma de México.

Pineda, F. A. (1986). El análisis interactivo de la adquisición del lenguaje. Revista Bibliográfica Trillas, Año IV, 17, 4-7.

Plaza, F. (1996). La disciplina escolar o el arte de la convivencia. Málaga, España: Aljibe.

Steinberg, L., Lamborn, S., Darling, N., Mounts, N. \& Dornbusch, S. (1994). Over-time changes in adjustament and competence among adolescents from authoritative, authoritarian, indulgent and neglectful families. Child Development, 65, 754-770.

Torres, L. E., Ortega, P., Reyes, A. y Garrido, A. (2008). Estudio de las interacciones diádicas. En O. Tena y $\mathrm{H}$. Hickman (Editoras) Avances de Investigación en Aprendizaje Humano. Desde lo básico hasta lo aplicado. México: Universidad Nacional Autónoma de México, FES Iztacala. Capítulo 5, pp. 115-140.

Vallejo, A. G. (2002). Estilos de paternidad y conflictos de autoridad entre padres y adolescentes totonacas en el medio rural. Tesis de Doctorado. México: Universidad Nacional Autónoma de México.

\section{ANEXO 1: DATOS SOCIOECONÓMICOS DE LA POBLACIÓN PARTICIPANTE EN LA INVESTIGACIÓN}

Para obtener la información socioeconómica de la población participante de este estudio se recurrió a la clasificación de la Asociación Mexicana de Agencias de Investigación de Mercado y Opinión Pública A. C. (AMAI). La información socioeconómica de dicha asociación está basada en estudios socioeconómicos por colonia y municipio. El municipio donde se encuentra ubicada la escuela donde se llevó a cabo esta investigación se localiza en la parte norte central del Estado de México, la colonia fue clasificada en el nivel $D$, según la AMAI, y tiene las características siguientes:

Nivel D: Está compuesta por personas con un nivel de vida austero y bajos ingresos. El jefe de familia de estos hogares cuenta en promedio con un nivel educativo de primara o secundaria, están empleados como obreros, personal de mantenimiento, vendedores de mostrador, choferes públicos, maquiladores, etc., y un gran número de madres de familia cuenta con estudios de primaria, se dedica al hogar y eventualmente al comercio informal.

Perfil del hogar: viven en inmuebles propios o rentados. Las casas o departamentos cuentan, en general, con una recámara, un baño, sala-comedor y cocina. La mitad de estos hogares tienen calentador de agua. Estas casas o departamentos son en su mayoría de interés social o de rentas congeladas (tipo vecindades). Los hijos realizan sus estudios en escuelas de gobierno. Las personas de este nivel suelen desplazarse por medio de transporte público y si llegan a tener auto es de varios años de uso. La mayoría de los hogares cuenta con un televisor, videocassettera y/o equipo modular de bajo 
costo. Se puede decir que prácticamente no poseen ningún tipo de instrumento bancario. Sus diversiones y pasatiempos suelen ser en parques públicos y esporádicamente en parques de diversiones. Suelen organizar fiestas en sus vecindades y tomar vacaciones una vez al año en excursiones a su lugar de origen o al de sus familiares. Su ingreso mensual familiar varía entre 1600 y 4000 pesos. 